\title{
APRESENTAÇÃO
}

\section{Interlocuções teóricas e a língua em uso: Linguística Sistêmico-Funcional, Linguística Enunciativa e Linguística Aplicada}

Rodrigo Esteves de LIMA-LOPES

(Universidade Estadual de Campinas - Instituto de Estudos da Linguagem - Departamento de Linguística Aplicada/Programa de Pós-Graduação em Linguística Aplicada - Campinas - SP - Brasil)

Lucia Rottava

(Universidade Federal do Rio Grande do Sul - Instituto de Letras - Departamento de Letras Clássicas e Vernáculas/Programa de Pós-Graduação em Estudos da Linguagem - Porto Alegre - RS - Brasil)

\section{As contribuições deste volume para os estudos Sistêmico- Funcionais}

Abordar o funcionamento da linguagem é olhar para seu uso e representa movimentos compreensivos diversos para que interlocuções teórico-metodológicas possam ser evidenciadas em pesquisas. Esses movimentos permitem que sejam observadas na teoria e na prática contribuições linguísticas para a formação docente e possíveis desdobramentos da pesquisa para compreender como a língua é usada nos mais diversos contextos e com propósitos analíticos distintos, além dos processos de ensino e de aprendizagem. 
Este volume da revista DELTA traz uma pouco dessa interlocução por seus autores entenderem que a complexidade é inerente à língua em termos de sua realização, instanciação, estratificação e de relações que constituem redes de significados (Halliday 2003; Matthiessen 2005). Esses atos de significação (Halliday 1993) ${ }^{1}$ se realizam em texto e este é tomado como ponto central para a discussão de uma variedade de conceitos abordados pela Linguística Sistêmico-funcional, Linguística Enunciativa e Linguística Aplicada.

Tal interlocução, necessariamente, passa pela percepção de que as diversas abordagens teóricas podem trabalhar de forma a criar diferentes ambiências analíticas, cada uma delas contribuindo para compreensão dessa complexidade. De forma geral, todos os artigos deste volume analisam questões teóricas e analíticas que pavimentam o caminho para a ampliação dos estudos pelos diferentes caminhos que a relação de pluralidade aqui proposta pode se desenvolver. A Linguística SistêmicoFuncional (doravante LSF) ${ }^{2}$ é o ponto de partida dessa discussão dada

1. Vale lembrar que no contexto brasileiro, estudos e pesquisas linguísticas sistêmicofuncionalistas tem um dos seus importantes pontos de ebulição na PUC/SP, graças ao trabalho pioneiro da $\operatorname{Prof}^{\mathrm{a}} \mathrm{Dr}^{\mathrm{a}}$ Leila Barbara. No início dos anos 70 , foi das fundadoras do Programa de Estudos Pós-Graduados em Linguística Aplicada e Estudos da Linguagem (LAEL) da PUCSP, integrante da ABRALIN (Associação Brasileira de Linguística) e da ASFAL (Associação Latino Americana de Linguística Sistêmico-Funcional). Nos anos 90 coordena o DIRECT, um projeto multisítio com sede na PUCSP, envolvendo diversas universidades brasileiras e estrangeiras. Este projeto foi um dos pioneiros na análise da linguagem profissional, sendo um centro de formação de diversos pesquisadores que hoje se destacam no contexto da Linguística Aplicada do Brasil. Nos anos 2000, iniciou o projeto SAL (Sistêmico Através das Línguas), também envolvendo diversas universidades Brasileiras e de toda a América Latina. Atualmente, Leila dedica-se a estudos centrados na área de Gramática Sistêmico-Funcional, com foco em usos de linguagem e gêneros relacionados à formação cidadã e para o trabalho, com especial ênfase na descrição da Língua Portuguesa. Atua principalmente na área da linguística aplicada viabilizando sua interface com a análise do discurso, linguagem dos meios de comunicação e linguagem do trabalho. É por sua contribuição a área, que dedicamos este volume à Leila Barbara.

2. Halliday foi um dos mais influentes linguistas a partir da segunda metade do século XX. Nascido em 1925 no Reino Unido, foi orientando de J. R Firth (1890-1960), uma importante influência em seu trabalho. Ele lecionou na Universidade de Londres e na Universidade de Sidney, esta última até sua aposentadoria. O trabalho de Halliday sempre esteve relacionado à compreensão de como a língua é estruturada para o uso, tendo dado a linguística o status de ciência social aplicada, uma vez que suas teorias versam sobre a indivisibilidade entre a linguagem, o significado e seu contexto. O trabalho de Halliday 
sua natureza social, sendo que as reflexões realizadas em seu espectro, neste volume da revista DELTA, são inclusas análises lexicogramaticais orientadas por Halliday e Matthiessen (2014), semântico-discursivas com base nos estudos de Martin e Rose (2003) e Martin e White (2005), e de gêneros e seu potencial pedagógico de acordo com Martin e Rose (2008) e Rose e Martin (2012). Temas também incluem a utilização de parsers para análise comparativa de tradução, metáfora gramatical, transitividade, análise de estrutura temática e avaliatividade.

Algo comum aos estudos sistemicistas é sua preocupação com contextos reais de produção e ensino, fazendo dela mais do que simplesmente um processo analítico desvendador do texto e seu discurso, mas criando uma linguística que seja aplicável na mudança e transformação social (Martin 2016). É esta preocupação discursiva que leva ao primeiro ponto de convergência, a Linguística Enunciativa orientada pela perspectiva Benveniste (1989 e 2014). Esta abordagem diz respeito ao texto e à escrita, visto que abordar o funcionamento da linguagem significa que "Amplas perspectivas se abrem para a análise das formas complexas do discurso [...]" (Benveniste 1989: 90). Tal interlocução leva a uma clara ampliação dos espectros analíticos, demonstrando como a pluralidade teórica pode contribuir para a compreensão do fenômeno da linguagem.

Outra interlocução importante oferecida por este volume está entre a LSF e o Interacionismo Sociodiscursivo (ISD). Tal ponto de contato parece se apoiar no fato de que ambas valorizam o processo comunicacional em ambiente social. Nas duas perspectivas, o processo é a utilização da linguagem em situações reais de comunicação, de forma a oferecer uma importante resistência a abordagens estruturalistas. Isso acaba por levar ambas as abordagens a se preocupar de forma bastante enfática com o contexto de produção e o conhecimento que o falante possui.

A tradução e a linguística do corpus também são temas que devem ser observados como importantes para um volume como este. Primeiramente, por que a tradução tem sido um constante tema de reflexão

Seu recente falecimento é uma perda inestimável para os estudos da linguagem e para o desenvolvimento de uma linguística que seja aplicável na construção de uma sociedade mais justa e igualitária. 
nos estudos sistemicistas (Halliday 2001; Yallop 2001), com diversos estudos realizados no contexto brasileiro (Rodrigues-Júnior 2005; Ventura 2000; Espindola 2016). Em termos gerais, tais trabalhos buscam ir além da pura equivalência estrutural no processo tradutório, refletindo sobre como elementos culturais são responsáveis pela transformação nos padrões de escolha. Algumas das reflexões propostas nesse volume extrapolam a abordagem funcionalista, mostrando como ela pode se relacionar com outras teorias, tais como a perspectiva integradora da tradução.

Já a linguística o corpus tem suas origens em um universo teórico similar à GSF (Stubbs 1996; Lima-Lopes 2017). Ambas nascem no bojo da linguística neo-firthiana e buscam o levantamento de padrões de escolha como elemento fundamental da compreensão da linguagem. Apesar de possíveis diferenças, as duas abordagens elegem o contexto como ponto de partida para a compreensão da linguagem e seu ensino. Suas ferramentas tem sido um importante ponto de apoio para os estudos sistêmicos e da tradução, pontos amplamente referenciados neste volume.

Os gêneros são hoje importante ponto de convergência na abordagem sistêmica. Tendo como base inicial os estudos de Hasan (Halliday \& Hasan 1991; Hasan 2014), eles passam a ser encarados como uma forma de tangibilizar os elementos presentes no contexto de cultura. Isso necessariamente levaria a encarar a cultura como um sistema de gêneros (Martin; Rose 2008), imbricados nas práticas sociais de cada realidade linguística. Aqui as contribuições estão na reflexão de como as constelações de gêneros do português se dão no contexto de seu ensino, observando-se tanto no contexto de língua materna como estrangeira para não-ouvintes. Uma reflexão similar se dá sobre observação de sistemas metafuncionais, em especial a transitividade e o sistema temático, cujas reflexões são insumo para reflexões sobre o ensino e sobre o sistema de avaliatividade.

O potencial desses estudos está na ressignificação de pressupostos teóricos e metodológicos que orientam reflexões que priorizam práticas de ensino e aprendizagem de línguas (Celani 1998) e de profissionais da linguagem. 


\section{As interlocuções propostas neste volume ${ }^{3}$}

Este número da revista DELTA está organizado em três blocos. O primeiro é composto por artigos que seguem uma abordagem lexicogramatical e semântico-discursiva; o segundo bloco engloba estudos relacionados ao gênero e seu impacto no processo de ensino e de aprendizagem da língua portuguesa com contribuições da e para a Linguística Aplicada, sendo o terceiro de estudos que estabelecem interlocuções cujo objetivo principal é ampliar o escopo dos estudos em GSF.

O primeiro bloco, denominado "Perspectivas analíticas, Corpus e LSF", organiza-se em torno de nove estudos que tem como temática parser, SLF e tradução, metáfora gramatical, transitividade, análise de estrutura temática e avaliatividade.

O primeiro artigo deste bloco é de autoria de Mike Scott da Aston University e apresenta um software para gerenciar downloads de texto de grandes bancos de dados online. Trata-se de uma ferramenta, de acordo com o autor, que ajuda, dentre outros recursos, a padronização de bases de dados com textos completos que permitem ao usuário pesquisar e, em seguida, visualizar e, idealmente, baixar o texto completo de artigos relevantes. A reflexão propiciada por Scott é importante por fornecer insumos para a organização de dados linguísticos em grande escala, caminho pelo qual a GSF se direciona a paços largos.

O segundo artigo de autoria de Rodrigo Esteves de Lima-Lopes, da Universidade Estadual de Campinas, discute escolhas de transitividade. O autor compara o conto "Amor", publicado pela escritora Clarice Lispector no livro "Laços de Família" em português, e sua tradução para o inglês. A análise contou com a utilização do software "UAM CorpusTool" (O'Donnell 2016) e da ferramenta online de alinhamento de corpora (YouAlign 2016). Os resultados apontam para uma reflexão

3. As discussões que levaram ao embrião deste volume começaram em abril de 2016 por ocasião do VI Encontro SAL (Systemics Across Languages) e no V Seminário de Formação Docente PIBID-Português, ambos simultaneamente realizados na Universidade Federal do Rio Grande do Sul. A ideia começou a circular nos corredores do evento e, o que inicialmente, era um projeto destinado à publicação dos trabalhos ali apresentados, ganhou corpo, expandiu-se e se tornou o conjunto de artigos que aqui apresentamos. 
sobre a equivalência de escolhas e à construção de sistemas textuais específicos, motivados por questões culturais da língua de chegada. De autoria de Adail Sebastião Rodrigues Júnior, da Universidade Federal de Ouro Preto, o terceiro artigo discute o papel das expansões para a constituição das identidades e dos traços de personalidade dos personagens da obra "O Retrato de Dorian Gray", de Oscar Wilde, e de sua tradução, feita por João do Rio, e adaptações, de Clarice Lispector e Cláudia Lopes, para o português brasileiro. O autor tem seu foco na configuração discursiva ou o eixo narrativo que desvela o ponto de vista do narrador, de forma a também problematizar os conceitos de omissão e reescrita no contexto da tradução literária e seus impactos culturais no(a) leitor(a) do texto-alvo. Os resultados de Lima-Lopes se Rodrigues Júnior apontam para a reflexão sobre a possível convergência entre a GSF, a Linguística do Corpus e os Estudos tradutórios.

De autoria conjunta de Lucia Rottava da Universidade Federal do Rio Grande do Sul e de Sulany Silveira dos Santos, da Universidade Estadual de Ponta Grossa, o próximo artigo analisa construções metafóricas no fluxo de informação em textos produzidos por alunos brasileiros ingressantes em contexto acadêmico. O estudo revela baixa ocorrência de construções metafóricas e, diante desse resultado, aponta caminhos para práticas didático-pedagógicas que visam ao letramento acadêmico.

O quinto artigo deste bloco é de autoria de Viviane Herbele da Universidade Federal de Santa Catarina (UFSC) destaca a relevância da Linguística sistêmico-funcional (LSF) para estudos em Linguística Aplicada e apresenta considerações gerais sobre LSF, o contexto de situação e as metafunções, para então discutir o sistema de transitividade na LSF. Como suporte teórico-metodológico para a análise de textos produzidos em diferentes mídias e contextos sociais, a LSF pode contribuir para a compreensão da linguagem como prática social multisemiótica em contextos educacionais.

O sexto artigo é de autoria de Luciane Sippert da Universidade Estadual do Rio Grande do Sul (UERGS) e aborda a estrutura temática em resenhas produzidas por alunos no Ensino Superior. O estudo mostra que os padrões de estrutura temática estão diretamente relacionados à composição dos gêneros e ao conhecimento que os alunos possuem 
em relação à lexicogramática, aos aspectos semântico-discursivos e estruturais do texto.

Estes estudos são relevantes por reafirmar a já intrínseca relação entre os estudos de GSF e o ensino de línguas.

Abordando a temática avaliatividade, Glivia Guimarães Nunes e Sara Regina Scotta Cabral, ambas da Universidade Federal de Santa Maria (UFSM), embasam-se no Sistema de Avaliatividade (Martin; White 2005) para abordar uma das categorias da atitude: o julgamento, que avalia o comportamento das pessoas segundo valores de estima social (capacidade, tenacidade e normalidade) e sanção social (propriedade e veracidade). As autoras mostram o predomínio de julgamentos negativos sobre o desempenho da Presidente, prevalecendo avaliações de estima social, sobretudo de capacidade. Essas avaliações são, em sua maioria, explícitas e indicam a interpretação de que a Presidente não está preparada para administrar o Brasil. Tal estudo reflete, logo, sobre a intrínseca relação entre os elementos avaliativos e os discursos politicamente motivados; área ainda pouco estudada no contexto da LSF no Brasil.

De autoria de Fabíola Aparecida Sartin Dutra Parreira Almeida da Universidade Federal de Goiás - Regional Catalão, o artigo apresenta uma análise do subsistema de atitude em comentários de participantes de dois blogs voltados para professores de inglês e das possíveis respostas de solidariedade apresentadas pelos interlocutores. Os resultados do estudo mostram certo alinhamento avaliativo entre os participantes do blog e as blogueiras por meio das escolhas dos elementos léxicogramaticais intensificados utilizados nas avaliações e nas respostas de solidariedade realizadas.

Para encerrar esse bloco, Osilene Maria de Sá e Silva da Cruz do Instituto Nacional de Educação de Surdos - INES/RJ realiza uma análise discursiva de resenhas elaboradas por graduandos surdos do curso de Pedagogia do Instituto Nacional de Educação de Surdos (INES). O trabalho revela também a importância do uso de estratégias adequadas de ensino de Língua Portuguesa escrita para tais aprendizes. 
O segundo bloco, constituído por cinco artigos, tem como temática comum os gêneros e sua relação com ensino e a formação do professor. Tais estudos mostram a clara interface desta teoria com a prática pedagógica em seu cotidiano, mostrando que a LSF é também uma importante ferramenta para seu desenvolvimento.

O primeiro artigo é de autoria de Estela Inés Moyano, da Universidad de Flores em Buenos Aires, Argentina e aborda o desenvolvimento da leitura e da escrita acadêmica e profissional em nível universitário. Em seu artigo, a autora reflete sobre questões teóricas de aprendizagem, além de discutir temas relacionados às demandas metodológicas e de gestão quando da aplicação de um programa de leitura e escrita de tal natureza.

Na sequência, Cristiane Fuzer, da Universidade Federal de Santa Maria (UFSM), delineia um percurso de leitura guiado por aspectos relevantes da teoria sistêmico-funcional que revelem a instanciação de gêneros da família das estórias. Focalizam-se as noções de estratificação, realização e instanciação da linguagem para esclarecer a noção de gênero na Linguística Sistêmico-Funcional e analisar realizações linguísticas que evidenciam o propósito sociocomunicativo e a estrutura esquemática de gênero numa amostra de textos socialmente designados como fábulas, muito frequentes no contexto escolar brasileiro.

Edna Cristina Muniz da Silva, da Universidade de Brasília, traz uma discussão a respeito de gênero e registro. A autora destaca que gêneros e registros são termos utilizados pelos sistemicistas para se referirem ao significado e à função da variação entre os textos na dimensão contextual, bem como a quanto os textos se assemelham e se distinguem por expressarem linguística e discursivamente traços do contexto social em que são utilizados.

Ainda abordando gêneros textuais, desta vez da família das estórias, Maria do Rosário da Silva Barbosa, da Universidade Federal de Pernambuco, discute a construção de gêneros da família das estórias produzidas por escolares e os resultados pontuados pela autora dizem respeito ao fato que as etapas de gêneros da família das estórias variam de acordo com o propósito e o contexto de cultura do aprendiz e influenciam nas escolhas linguísticas - lexicais e gramaticais. 
Por fim, o artigo de Orlando Vian Jr., da Universidade Federal de São Paulo, explora os multiletramentos. O autor visa entender como os multiletramentos, a partir de uma perspectiva sistêmica e complexa, devem estar presentes na formação de professores de línguas e, mais especificamente, em seu Conhecimento Sobre a Língua(gem) (CSL). Seu argumento central de que a noção de conhecimento atualmente em uso na área de formação de professores requer revisões, ampliações e reorientações em seu escopo com vistas a uma educação linguística mais eficaz e realista, requerendo discussões sobre a formação do professor de línguas e a construção de seu conhecimento no âmbito dos cursos de graduação em Letras.

Encerrando este volume, há um conjunto de artigos que se constituem interlocuções com teorias linguísticas diversas.

O primeiro dos artigos é de autoria de Aliny Sousa Mendes, Wagner Rodrigues Silva, ambos da Universidade Federal do Tocantins, e de Paulo da Silva Lima, da Universidade Federal do Maranhão. Os autores apresentam um estudo comparativo entre as abordagens teóricas desenvolvidas no Interacionismo Sociodiscursivo (ISD) e na Linguística Sistêmico-Funcional (LSF) visando à identificação de semelhanças e/ ou diferenças entre essas duas correntes teóricas, visto que ambas têm contribuído significativamente para os estudos linguísticos desenvolvidos no Brasil, seja no campo teórico ou aplicado.

O segundo artigo deste bloco é de autoria de Valdir do Nascimento Flores da Universidade Federal do Rio Grande do Sul e apresenta uma proposta de interpretação para a expressão enunciação escrita, presente em "O aparelho formal da enunciação", de Émile Benveniste, com vistas ao estabelecimento de princípios norteadores do estudo da enunciação escrita no quadro formal de realização da enunciação.

Carmem Luci da Costa Silva da Universidade Federal do Rio Grande do Sul aborda o conceito de texto em uma perspectiva enunciativa de linguagem. O pressuposto do artigo é de que, a cada vez que a língua atualizada em discurso, via enunciação, há uma nova experiência de significação instaurada na linguagem. 
O quarto artigo deste bloco é de Cleci Regina Bevilacqua da Universidade Federal do Rio Grande do Sul e apresenta as definições de traduções, os princípios e elementos implicados no processo tradutório a partir das propostas de duas autoras, fundamentais nos Estudos de Tradução: Christiane Nord, que segue uma perspectiva funcionalista, e Amparo Hurtado Albir, que defende uma perspectiva integradora da tradução, identificando os elementos de aproximação entre ambas.

Para encerrar este volume, as autoras Laura Rampazzo do Instituto Federal de São Paulo e Solange Aranha, da Universidade Estadual Paulista "Júlio de Mesquita Filho" de São José do Rio Preto, concentramse no contexto de Teletandem, um ambiente virtual e colaborativo de aprendizagem de línguas em que estudantes universitários se encontram semanalmente por meio de ferramentas de comunicação síncrona para a aprendizagem da língua um do outro.

Recebido em: 01/08/2017

Aprovado em: 10/10/2017

e-mail:r11307@unicamp.br e-mail: luciarottava@yahoo.com.br

\section{Referências bibliográficas}

Benveniste, E. 1989. Problemas de linguística geral II. Tradução de Eduardo Guimarães [et al]. Campinas/SP: Pontes. . 2014. Últimas aulas no Collège de France 1968 e 1969. Tradução de Daniel Costa da Silva [et al]. São Paulo: Editora Unesp.

Celani, Maria Antonieta. 1998. Transdisciplinaridade na Linguística Aplicada no Brasil. In: Signorini, Inês; CAVAlCAnti, Marilda (Orgs.). Linguística e Transdisciplinaridade. Campinas: Mercado de Letras. p. 129-142.

EsPINDOLA, E. 2016. A systemic functional translation analysis of thematic structure: directing attention to Yoda's linguistic manifestation. Word, 62.1: 22-34. Doi: 10.1080/00437956.2016.1141938.

HallidAY, M. A. K. 2001. Towards a theory of good translation. In E. Steiner \& C. Yallop (Eds.). Exploring Translation and Multilingual Text Production: Beyond Content. Berlin, Boston: DE GRUYTER. p. 13-18. 
. 1993a. The act of meaning. Georgetown University Round Table on Languages and Linguistics 1992: language, communication and social meaning 7-21. Washington, DC: Georgetown University Press.

2003. Introduction: On the "architecture" of human language. In J.J. Webster (ed.) Collected Works of M.A.K. Halliday, Volume 3. London \& New York: Continuum. p. 1-35.

Halliday, M. A. K., \& Hasan, R. 1991. Language, context and text: aspects of language in a social-semiotic perspective. Oxford: Oxford University Press.

Halliday, M.A.K.; Matthiessen, C.M.I.M. 2014. Halliday's Introduction to Functional Grammar. 4. ed., Milton Park, Abingdon, Oxon: Routledge.

HASAN, R. 2014. Towards a paradigmatic description of context: systems, metafunctions, and semantics. Functional Linguistics, 1.1: 1-9.

Lima-Lopes, R. E. de. 2017. Reflexões sobre as possíveis contribuições da Linguística do Corpus para a Gramática Sistêmico Funcional: Transitividade e Classificação de Processos. Caletroscópio, 5.9: 9-25.

Martin, James R;. 2016. Meaning Matters: A Short History of Systemic Functional Linguistics. Word 62.1: 35-58.

Martin, James R.; Rose, D. 2008. Genre Relations: Mapping Culture. London and Oakville: Equinox.

Martin, James R.; White, Peter R.R. 2005. The language of Evaluation. London: Palgrave.

Matthiessen, Christian M.I.M. 2005. "The "architecture" of language according to systemic functional theory: developments since the 1970s.” In: Ruqaiya Hasan, Christian M.I.M. Matthiessen \& Jonathan Webster (eds.). Continuing discourse on language. Volume 2. London: Equinox. p. 505-561.

O’DonNell, M. 2016. UAM CorpusTool - Versão 3.3. Recuperado de http://www.corpustool.com

Rodrigues-JÚNIOR, A. S. 2005. An Exploratory Study of Representation of Gay Characters in a Parallel Corpus of Short Stories: a SystemicFunctional Approach. Cad. Tradução, 16, 83-104.

Rose, D.; MARTIN, J. R. 2012. Learning to write, reading to learn. Londres: Equinox.

StubBs, M. 1996. British Traditions in Text Analysis: Firth, Halliday and Sinclair. In M. Stubbs, Text and corpus analysis. London: Blackwell. p. $22-50$. 
Ventura, C. S. M. 2000. Análise Temática em Estudos de Tradução: o Caso dos Relatórios Anuais de Empresas Brasileiras. Dissertação de Mestrado em Linguística Aplicada ao Ensino de Línguas - LAEL. Pontifícia Universidade Católica de São Paulo - PUCSP.

YALLOP, C. 2001. The construction of equivalence. In E. Steiner \& C. Yallop (Eds.). Exploring Translation and Multilingual Text Production: Beyond Content. Berlin, Boston: DE GRUYTER. p. 229-246.

YouAlign. 2016. Recuperado 3 de novembro de 2016, de http://www. youalign.com/ 